\title{
Principios teóricos y metodológicos de los estudios métricos de la información
}

\author{
Salvador Gorbea-Portal \\ Investigadorinvitado del Cen tro Unversitario de \\ Investigaciones Bibliotecológicas, UNAM. Circuito \\ In te rior, Torre II de Hu ma ni da des, piso 12, Ciu dad \\ Uni ver sitaria, C. P. 04510. Tel. 56-23-03-60 \\ e-mail:portal@servidor.unam.mx
}

\section{RESUMEN}

Se presen ta un mar co teó ri co con cep tual so bre los as pec tos me to do ló gi cos y las re lacio nes interdisciplinarias de los Estudios Métricos de la Información, a través de las disciplinas que con di cionan el surgi mien to de las de nomina das es pecia li da des métricas de la in forma ción y de la pro pues ta de un es que ma en el que se re la cio nan aque llos ele men tos que, des de el punto de vis ta me to do ló gi co, pue den ser con si de ra dos en este tipo de es tu dio, in clu yen do aquellos re lacionados con el análisis de sus variables.

\section{ABSTRACT}

The article considers the theoretically conceptual framework of methodological aspects and tho se per tai ning to the in ter dis ci pli nary links of In for ma tion Me tri cal Stu dies by means of disciplines conditioning the emergence of the now-designated metric specialties of information and the proposal of a sche me to re la te ele ments which, from a metho do lo gi cal viewpoint, $m$ ay be con side red in stu dies of this na tu re, in clu ding tho se re la ted to the analysis of theirva riables.

\section{INTRODUCCION}

$L$ asactividades bibliotecaria, archivística, bibliográficaycientífico-informativaduran te su sur gi mien to y de sa rrollo han ido conformandoun sis te ma de conocimien tos, in tegrado por disciplinas que comprenden a su vez mé to dos, téc ni cas y mar co teóri co propio que res ponden a su ob je to y tema de es tu dio.

Sin embargo el camino que han recorrido en la conceptualización de disciplinas propias, propiciadofundamentalmen te porelfenómeno de in te gra ción y di fe ren cia ción de la ciencia, no ha estado exento de controversia; entendido este fenómeno como un proceso nece sario y carac te rís ti co del de sa rrollocientífi co-téc ni co de nues tro tiem po que se ma nifies ta en to das las dis ci pli nas cien tífi cas y ramas del saber.

Como consecuencia de este proceso surge y se ge ne ra li za en la Eu ro pa delEste, du ran te la dé ca da de los 60, la de no mi na ción de una disci pli na que era en ten di da en mu chos paí ses y au to res con pre ten siones glo balizado ra de todas las anteriores actividades en tanto otros reconocían en este térmi no elsurgimien to de una nueva disciplina, denominada por A.I. Mikhailov, A.I. Chernii y R.S. Guiliarevkii, (1) sus autores, como "Informática", quienes reconocían que aún le faltaba desarro llo para ser tra ta da como tal.

Procesos similares de integración o diferencia ción die ron ori gen, en otras re gio nes y piases, a otros tér mi nos con el in te rés de de fi nir disciplinas tales como: Documentación o Cien cias de la Do cu men ta ción, Cien cia de la Información, Archivología, Bibliografía o Bibliogra fología, en tre otras. Mien tras tan to, laBibliotecologíacontinuaba su desarrolloy consolidación comodisciplinacientífica,mediante las relaciones interdisciplinarias entre estas nuevas disciplinas y otras de las Ciencias técnicas, Sociales y Matemáticas.

La historia del desarrollo del conocimiento científico nos enseña que en ocasiones una gran dis ci pli na pue de dar lu gar a un con jun to deespecialidades con el mis mo ob je to de estu dio, o de dis ci pli nas cien tíficas que, aun que muy relacionadas con la primera, tienen su marca da di fe ren cia en cuan to a su pro pio objeto y tema de estudio, dando lugar por sus propias relaciones y naturaleza a un sistema de conocimientos científicos.

Autores como A. Zokolov ${ }^{(2)}$ han aportado claridad sobre este fenómeno al reconocer comodis ci plinas cien tíficas a la Bibliote cología, la Archivología, la Bibliografología y la Ciencia de la Información, así como el nivel de independencia que existe entre ellas, enmarcándolas como disciplinas que pertenecen al Com ple jo Bi blio ló gi co de las Cien cias Comunicativas Informativas.

Este pun to de vis ta, plan tea do por Zo kolov, es re to ma do porE. Se tién y S.Gorbea, ${ }^{(3)}$ quie nes también re co nocenel niveldein de pendencia e interrelaciones que se da en este grupo de dis ci plinas y las en mar can den trodelSistema de Conocimientos Científicos Bibliológico-Informativo. 
Paralelamente a este fenómeno, se observa otro de los ras gos dis tin ti vos del de sa rrollo de la cien cia ac tual, lo cons ti tu ye, sin lu gar a dudas, una cre cien te pe ne tra ción de los mé to dos y modelos matemáticos y estadísticos en los dis tin tos cam pos de la cien cia, como re sul tado de la in terdisciplinariedadqueocasionael propiodesarrollocien tífi co de nues tros tiempos, as pec to que es se ña la do por J. M. Ló pez Piñero en su obra. ${ }^{(4)}$

Esta in te rre la ción pro pi cia la ten den cia de un fenómenoconocidocomola ma te ma ti zación de las ciencias, lo que provoca el surgimientos de especialidades métrica tales como la Econometría, la Biometría, la Psicometría, en tre otras; es de cir, la apli ca ción de los mé todos y modelos matemáticos en forma reproduc ti va, a las cien cias econó mi cas, bioló gi cas y psicológicasrespectivamente.

El Sistema de conocimientos científicos Bibliológico-Informativo cuyas disciplinas se relacionan anteriormente, no ha estado ajeno a este fenómeno; es por ello que al igual que en otras ramas del conocimiento, en ésta los problemas relacionadoscon el es tu dio de las cantidades de documentos científicos y las magnitudes, características y regularidades de los flujos de información documentaria han sido abor da dos en for ma in ten si va des de finales de la década del 60.

Conside rable ha sido la produc ción científica que se ha ge ne ra do en ma te ria de es tu dios métri cos de la in for ma ción, tal que ha per mi ti do la compilación de varios índices bibliográficos, en tre los cua les se pue den des ta car: el de A. Prichard y G. Wittig ${ }^{(5)}$ quienes publican en 1981 una bibliografía de trabajos sobre este tema, que cu bre el pe río do com pren di do en tre 1874 y 1959 e in clu ye unas 600 pu blicaciones; los de R. Hjer ppe, quien publi ca un índice bibliográfico sobre el tema en $1980^{(6)}$ y que contiene 2032 referencias bibliográficas y su su ple men to con 518 re fe ren cias apa re cido en 1982 en la revista Scientometrics ${ }^{(7)}$ y más recientemente en 1991 A. Khurshid( ${ }^{(8)}$ y H.Sahai publican una bibliografía selectiva so bre las Le yes y dis tribu ciones mé tri cas más im portan tes en esta te mática.

Otro es fuer zo que vale des ta car se en este senti do es el que vie ne rea li zan do A. Schu bert, ${ }^{(9)}$ quien des de 1982 y con cier ta sis te ma ti cidad, publica en la revista Scientometrics una biblio gra fía con los úl ti mos tra ba jos que apa recen sobre el tema.

Es precisamen te el com por ta mien to de la produc ción cien tífica ge ne ra da so bre el tema, en la que se identificantrabajos de cor te teórico y aplicaciones prácticas, así como el surgimiento y tratamientoterminológicoque desde la dé ca da de los 40 se le ha dado a este tipo de es tu dio con el pro pó si to de iden ti fi car disci pli nas y es pe cia li da des, lo que le per mi tióa E. Se tién y S. Gor bea ${ }^{(10)}$ pro poner la iden tificación de un conjun to de es pecialidades métri cas aso cia das a las dis ci pli nas que in te gran ellla ma do Sis te ma de Co no ci mien tos Cien tí ficos Bibliológico-Informativo.

No obstante a esta producción científica genera da, to da vía per sis te una gran con tro versia en las definiciones y relacionesinterdisciplina rias de los dos ni ve les de es truc tu ra del conocimiento en esta rama del saber: el primer nivel referido a las dis ci plinas cien tíficas antes se ña la das y el se gun do al de las es pe cia lidades métricas que han surgido como consecuencia de la aplicación de métodos y modelos matemáticos y estadísticos a las referidasdisciplinas.

Es por todo ello que el presente trabajo, sin pretender dar solución al problema controversial en torno a los conceptos y la diversidad de tra ta mien tos eti moló gi cos, tie ne como objetivo presentar un posible enfoque del problema des de el pun to de vis ta del sis te ma de conocimiento que integran las disciplinas que dieron origen a esta red de especialidades, en las que sin lu gar a du das se apor tan estu dios y resul ta dos de in ves ti ga ción con objetivos, fines y propósitos diferentes que en ri que cen ellen gua je for mal de varias dis ci plinas científicas.

Es objetivo también de este trabajo reflexionar sobre los aspectos metodológicos en los estudios métricos de la información, através de la pro pues ta de un es que ma en el que se relacionan aquellos elementos que, desde el punto de vis ta me to do ló gi co pue den ser considerados en este tipo de estudio, incluyendo aquellos relacionados con el análisis de las va ria bles se gún los fi nes que co mún men te se persiguen en cada trabajo.

\section{ANTECEDENTES Y TER MINOLOGIA EM PLEA DA EN LOS ES TUDIOS ME TRI COS DELA IN FORMA CION.}

Losantecedentes de los estudios métricosde la información han sido tratados en varias ocasiones por diferentes autores, tales como O. Nacke ${ }^{(11)}$ y L. Re don do, I. Gar cía, L. Es pinosa, ${ }^{(12)}$ am bos en 1979 y ci ta dos por M. Morales en 1985.(13)

Estos trabajos coinciden en señalar que este tipo de estudio tiene sus antecedentes en 1896 , cuan do F. Cam bell(14) pro pu so por primera vez explorar la información por temas. Varios autores, entre ellos el propio Nacke, reconocen la presencia de estudios similares rea li za dos en 1917 por F. J. Cole y Ne llie B. Eales, en la revista Science Progress, ${ }^{(15)}$ los cuales fueron denominados como "Análisis Estadístico de la Literatura”.

Posteriormente E.W. Hulme, en su libro publicado en $1923^{(16)}$-citado también por Nacke-, pre di jo una nue va lí nea para el es tudio de los procesos de información. Hulme fue el primero en emplear el término de "Bibliografía Estadística" (Statistical Bibliography) aplica do a los proce sos es ta dís ti cos y tec no ló gi cos den tro de la co mu ni ca ción científica, determinada por los conteos documentarios.

Más adelante señala el propio Nacke en su tra ba jo que, en 1948 S.R. Ran ga nathan, al comentar una ponencia de J.D. Bernal en la Conferencia de ASLIB, empleó el término "Librametry"(17). Este término ha sido empleado por M. Morales en $1988^{(18)}$ y por E. Setién y S. Gorbea en $1990^{(10)}$ en español como Biblio te co me tría aun que con ob je ti vos diferentes en cada trabajo; en éste último se de fi ne como "la apli ca ción de mé to dos y modelos matemáticos al estudio de los fenómenos pro pios de la actividad biblio te ca ria, con el fin de caracterizar el comportamiento de los componentes que integra esa actividad y las tendencias que se presentan en el uso de las biblio te cas y sus fon dos".

A. Prit chard en 1969 em pleó el tér mi no "Bibliometrics" (Bibliometría) ${ }^{(19)}$ por primera vez, para de no tar una dis ci pli na que la de fine como "la apli ca ción de los mé to dos ma te máti cos y es ta dís ti cos a los li bros y otros me dios decomunicación"

En España el autor J.M. López Piñero en $1972^{(4)}$ publica una de las obras más importan tes, es cri ta en es pa ñol, so bre el tema y la titula "El Análisis estadístico y sociométrico de la literatura científica".

En 1979 O. Nacke, en su trabajo citado an teriormente, ${ }^{(11)}$ en la en ton ces Re pública Fe deral Ale ma na-apo ya do en el Análi sis de 589 publicaciones en las que se aplicaban varios mé to dos y mode los ma te má ti cos a la solu ción de los problemas de la información-fue el primero en definir una nueva disciplina métrica de la información denominada como "In for me tría", al pa re cer aso cia da a la na ciente Cien cia de la In for ma ción.

Otro im por tan te tex to ve la luz en Ban ga lo re, In dia en 1983, don de Inna Ke da ge Ra vi chandra Rao, del Do cu men ta tion Re search \& Training Centre, Indian Statistical Institute, publica bajo el tí tu lo "Quan ti ta ti ve Methods for Library and in for mation Scien ce" (20) un li bro con un fuerte componente de Estadística en sus nue ve pri me ros ca pítu los, mis mo que más tarde es traducido al portugués en Brasil en 
1986 con el título Métodos Quantitativos em Biblioteconomia e Ciencia da Informaçao.

Más re cien te men te, en Bél gi ca, los au to res L. Egghe y R. Rousseau en 1990 pu bli can un libro titulado "Introduction to informetrics: Quantitative methods in library, documentation and information science."(21) Esta obra, editada por Elsevier, constituye un valioso texto en el que se ex pone el de sa rrollodelos métodos y modelosmatemáticos aplicados a las actividades bibliotecaria e informativa; posee un alto rigormatemático y presentaun ex ten so Análi sis de la biblio gra fía dis ponible sobre el tema has ta ese mo men to.

Variados han sido los conceptos y las definiciones em plea das en los es tu dios mé tri cos de la in for ma ción; ello co rro bo ra el cre ci mien to de in ves ti gaciones so bre el tema y la di versidad de en fo ques y pun tos de vis ta re la ti vos a laim bricación que se es table ce en tre el obje to y tema de es tu dio de es tas es pe cia li da des métri cas con el de las dis ci pli nas cien tífi cas que le die ron origen.

En este sen ti do vale des ta car la es tre cha re lación que guarda la Cienciometría, definida por Nali mov y Mulc sen koen $1969^{(22)}$ y ci tados por R. San cho(23) "como la aplicaciónde métodos cuantitativos a la investigación sobre el de sa rro llo de la cien cia como un pro ceso informativo".

La an te rior de fi ni ción of re ci da por los re fe ridos au to res nos aler ta que esta dis ci pli na métrica, perteneciente a la Cienciología, abarca en su tema de es tu dio todo lo con cer nien te al Análi sis cuan ti tati vo de los sis te mas y procesos cien tí fi cos, o sea, in clu ye la me tría no sólo de sus resultados (entre ellos las publicaciones científicas), sino tam bién de aque llos recursos de entrada que requiere el proceso científico para su desarrollo.

Tomando como punto de partida el análisis que so bre los mo de los eco nó mi cos de cos tobeneficio o inversión-resultado realiza $R$. Sancho en el trabajo antes citado, ${ }^{(23)}$ podemosiden ti ficar los ele men tos de en tra da y sali da que ope ran en el sis te ma cien cia-in ves tiga ción-de sa rrollo, pero en esta oca sión como procesoinformativo.

En el referido análisis, la autora distingue la entrada de recursos informativos, materiales y humanos, que condicionan la salida de recursos hu ma nos de alto ni vel, bie nes y co noci mien to que se tras mi te a tra vés de los de nominados canales de información formal (artículos, libro, pa ten tes, in for mes, etc.) y los informalesreferidosalasrelaciones in terperso na les que se dan en el pro ce so cien tí fi co.

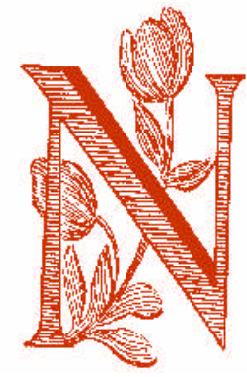

De ahí que cuan do en un es tu dio cien cio mé trico se plantea el aná li sis de uno de los re sul tados delde sa rrollo del procesocientífico, como por ejemplo la metría del conocimiento, se hace evidente que por constituir las publicacio nes cien tífi cas uno de los ca na les más uti lizados en la trans misión de conocimien to, este es tu dio re quie ra del uso de in di ca do res bi bliomé tri cos e in for mé tri cos, por el tipo de va riables documen ta les quees tos es tu dios emplean.

No obstante a que en este tipo de estudio se necesita de indicadores relacionados con el Sistema de Conocimientos Científicos Bibliológi co-In formativos, es decir, de la in formacióndocumentaria, no por ello sus objetivos están dirigidos a enriquecer este sistema de conocimientos, sino que éstos apuntan a ana li zar el com por ta mien to del sis te ma ciencia-investiga ción-desarrolloy es esta orien tación la que de ter mi na el fin úl ti mo de sus objetivos y por consiguiente a la disciplina que aportan el nuevo conocimiento es a la Cienciología.

"Variados han sido los conceptos y las definiciones empleadas en los estudios métricos de la información; ello corrobora el crecimiento de investigaciones sobre el tema y la diversidad de enfoques y puntos de vista relativos a la imbricación que se establece entre el objeto y tema de estudio de estas especialidades"

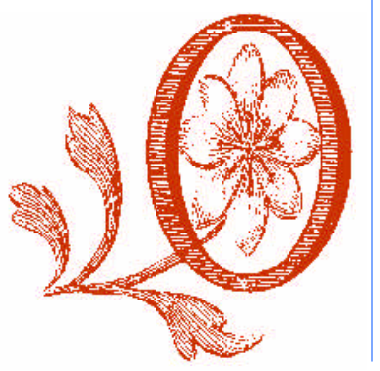

Es por todo ello que pode mos afir mar que en ocasiones algunos autores le atribuyen erróneamente a la Cienciometría funciones de disciplina rec to ra de los es tu dios mé tri cos de la información lo que acarrea consigo, por una par te, de li mi tar el ob je to de es tu dio de la Cien cio me tría a la me tría del co no ci mien to y, por otra, pen sar que la me di ción de una de las regularidades deldesarrollocien tífico, elcrecimiento ace le ra do de la cien cia, pue da en riquecer directamente el sistema de conocimientos científico Bibliológico-Informativo y no el de la Cienciología.

\section{B I B LIO-, CIEN CIO-, IN F OR-, ME TRIA?. CRISIS ETIMOLOGICA}

En la Segunda Conferencia Internacionalsobre Bibliometría, Cienciometría e Informetría de 1989 celebrada en Ontario, Canadá, B.C. Brookes ${ }^{(24)}$ reflexiona sobre el uso de los prefijos Biblio-Cien cio-, In for-aso cia dos a la raíz me tría y para ello acu de a los oríge nes de las disciplinas que propiciaron el surgimien to de cada uno de es tos tér mi nos en Euro pa y Es ta dos Uni dos.

Brookes en este tra ba jo re co no ce a la Bi bliometría limitada a la actividad bibliotecaria aunque ésta, señala, se debe enriquecer mediante las relaciones interdisciplinarias con es ta dís ti cos para re fi nar sus téc ni cas - as pec to éste que des de nues tro pun to de vis ta es vá lido para todas las especialidades métricas-. Más adelante reconoce que la Cienciometría ha llegado a ser muy productiva en los estudios so bre política cien tífica. 
Alreferirse al tér mi no In for me tría lo considera como el término más apropiado para enfrentar los nuevos desafíos tecnológicos que debe en fren tar en nues tros tiem pos la Cien cia de la In for ma ción, como por ejem plo el sur gimiento de las revistas electrónicas.

Has ta aquí apo yo ta les plan tea mien to en la medi da que di fe ren cian e iden ti fi can la pre sen cia de tres contenidos asociados a tres términoso especialidades métrica diferentes; sin embargo, en el primer planteamiento, al asociar la Bibliometría a las Bibliotecas, sería bueno destacar que en la Biblioteca contemporánea se distinguen cada vez con mayor énfasis dos actividades, la puramente Bibliotecaria y la Bi blio grá fi ca a la cual en Eu ro pa del Este se le asocia una nueva disciplina que se denomina Bibliografología o en otras regiones prefieren con ti nuar con el uso del tér mi no Bi blio gra fía.

En este sen ti do y re to man do la de fi ni ción origi nal de A.Pri chard en 1969 so bre Bi blio metría-"la apli ca ción de los mé to dos ma te má ticos y estadísticos a los libros y otros medios de comunicación"-ve mos más re laciona doel uso de este tér mi no a la Bi blio gra fía o Bi bliografo lo gía por de dicarseésta a la ac ti vi dad bi blio grá fica, es de cir, a la es truc tu ra y pro piedades de la información bibliográfica, uti li zan do para ello cual quier so por te o me dio formal de comunicación científica.

Por otra par te se ría opor tu no en esta cri sis etimológica poder reivindicar el término propuesto por Ranganathan y citado anteriormente "Librametry"-como la equivalencia al término de Bibliotecometría- y cuya definición se ofrece an te rior men te en el pun to 2 de este trabajo, para hacer referencia a los estudios cu yos resul ta dos con tri bu yen al de sarrollo de la actividad bibliotecaria, en tanto caracterizan el comportamiento de los com ponen tes que in te gran esa ac ti vidad y las tendencias que se presentan en el uso de las bibliotecas y sus fondos.

En este mis mo sen ti do pu die ra con side rar se a la Ar chi vo me tría como la apli ca ción de mé todos y modelos matemáticos y estadísticos al análisis y comportamiento de los documentos o ma nus cri tos de ar chi vos con el in te rés de identificar el comportamiento de fenómenos históricos asociados a la estructura y organiza ción de este tipo de fon do y do cu men to, cuyos re sul ta dos en ri que cen la or ga ni za ción de la ac ti vidad archi vís ti ca y por con si guien te la disciplina de Archivología.

La Cien cio me tría, como se ña la Broo kes en la cita anterior, es más productiva en estudios que apor ten ele men tos so bre po lítica cien tífica y est más relacionadaa la organización de la Cien cia y de los Sis te mas Cien tífi cos, aunque para ello ten ga que acu dir a va ria bles e in- dicadores documentarios, en algunas ocasiones, para comprobar uno de los indicadores que definen el crecimiento de la Ciencia, es decir, la generación de informaciónconocimiento.

Sire la ciona moses tos tér mi nos que de fi nen y conforman una red de especialidades métricas como descriptores de un tesauro y determina mos sues truc tu ra je rar qui za da, bien podemos conformar un esquema cuyas relacionespueden ser las siguientes:

\begin{tabular}{|c|c|c|}
\hline $\begin{array}{l}\text { Término } \\
\text { Genérico }\end{array}$ & TG & $\begin{array}{l}\text { - ES TU DIOS ME TRI COS DE LA } \\
\text { INFORMACION }\end{array}$ \\
\hline \multirow{3}{*}{ Usado por } & \multirow{3}{*}{ UP } & $\begin{array}{l}\text { ANALISIS ESTADISTICO DE LA } \\
\text { LITERATURA }\end{array}$ \\
\hline & & $\begin{array}{l}\text { ANALISIS ESTADISTICO Y SO } \\
\text { CIO ME TRI CO DE LA LI TE RA TU } \\
\text { RACIENTIFICA }\end{array}$ \\
\hline & & BIBLIOGRAFIAESTADISTICA \\
\hline \multirow{4}{*}{$\begin{array}{l}\text { Términos } \\
\text { Específicos }\end{array}$} & \multirow[t]{4}{*}{ TE } & ARCHIVOMETRIA \\
\hline & & BIBLIOMETRIA \\
\hline & & BIBLIOTECOMETRIA \\
\hline & & INFORMETRIA \\
\hline $\begin{array}{l}\text { Término } \\
\text { Relacionado }\end{array}$ & TR & CIENCIOMETRIA \\
\hline
\end{tabular}

En tal es truc tu ra no ten dría mos du das que nos con duz can a una cri sis eti mo ló gi ca o de contenido de cada una de ellas ni que propicie pen sar que una de las es pe cia li da des métricas relacionadas como términos específicos se pudieran superponer a los contenido de las otras, como en algunas ocasiones que se le atri bu ye a la In for me tría o a la Cien cio me tría disciplinasintegradoras del resto.

La revista Scientometrics, en su número 2-3 de 1994, publica un gru po con si de rable de artícu los que gi ran en tor no a un trabajo presentado por W. Glanzel y U. Schoepflin en la Cuarta Conferencia sobre Bibliometría, Informetría y Cienciometría, celebrada en Berlín en sep tiem bre de 1993 y que fue ra pu blicado en su totalidad en la revista y número de referencia. ${ }^{(25)}$

En éste artículo, que sirve como punto de la con troversia de sa ta da del res to de las críticas, los au to res re co no cen que hay un cre ci mien to acelerado en esta rama del saber y el interés por los indicadores cienciométricos también est en ascenso; sin embargo plantean que la Cien cio me tría est en crisis.

En tre las cau sas que les atri bu yen a la exis tencia de esta cri sis se en cuen tra la pér di da de un nú cleo im por tan te de cien tífi cos en esta es pecia li dad, y la baja ca li dad en el ma ne jo de las ba ses de da tos, en tre otras. A lo que pro po nen un es fuerzo parain te grare in ter disciplinarestudios de investigación, metodologías, programas de estudios, así como la creación de un có di go de éti ca.

De los 29 ar tícu los pre sen ta dos como con trover sia, 11 no es tán de acuer do con el plan teamien to de Glan zel y Schoepflin en que exis ta una crisis, 12 apoyan los planteamientos de és tos au to res, 2 los apo yan pero con re servas de con si de rar la mag ni tud de la cri sis a ni veles tan exa ge ra dos y 4 no dan cri te rios ni a favor ni en contra, sino que aportan elementos para sustentar nuevos criterios en torno al pro ble ma; tal es el caso de M. Krauskopf, (26) que propone un nuevo término, la Epistetometría, como el nombre que debe llevar esta disciplinabasa do en el ob je ti vo que ésta tie ne en la sociedad latinoamericana.

El análisis del artículo de Glanzel y Schoepflin y de las crí ti cas al que fue so me tido co rro bo ran la ne ce si dad de abrir el de ba te con el ánimo de formar es cue la en los as pectos teóricos que entrañan los estudios métricos de la información, aspecto éste que también M. Bonitz ${ }^{(27)}$ lo plantea en su crítica como una de las razones por las cuales est a favor de los planteamientos de Glanzel y Schoepflin.

Sin embargo considero, al igual que R. Rosseau, ${ }^{(28)}$ L. Egghe, ${ }^{(29)}$ entre otros, que la especialidadno est en crisis y que el artículo de los re fe ri dos au to res, no obs tan te, a que es bien recibido para un debate en este sentido, exagera el comportamiento de un fenómeno que ha es ta do pre sen te en to das las ra mas del saber y al igual que H. Eto ${ }^{(30)}$ soy partidario de iden ti ficarel fe nó me no no como una cri sis sino como con se cuen cia del ni vel de de sa rrollo en que se en cuen tran este tipo de es tu dios.

A nuestro criterio la crisis, tal como plantea A. Bookstein, ${ }^{(31)}$ no es tan gran de en la o las disciplinas, como en la aca de miaen ge neral, es decir en los estudiosos sobre el tema que tratan de agrupar un sistema de conocimientos sur gi do de va rias dis ci pli nas cien tíficasen una sola especialidad o disciplina métrica, ob vian do para ello que en este tipo de es tu dio intervienen diferentes objetos de estudios, propios de diferentes disciplinascien tíficas y por consiguiente utilizan también diferentes uni da des de ob servación.

Regresando a los planteamiento de A. Bookstein, ${ }^{(31)}$ coincido en que se hacen esfuer zos para to mar ven ta jas del mo men to y la pre sen cia de una Aso cia ción Profe sional para los Estudios Métricos de la Información, la cual constituye el medio más apropiadopara la "tor men ta de ideas" que per mi ta es cla re cer y arribar a con cep tos y de fi ni cio nes que con- 
tri bu yan al escla re ci mien to de un mar co teórico-conceptual e interdisciplinario que siente la base del futuro de los estudios métricosde la in for ma ción. As pec to éste que no se re suelve con la pre sen cia de un có di go de éti ca, pues como bien señala W. E. McGrath ${ }^{(32)}$ el có digo de éti ca va más di ri gi do a re sol ver problemas relacionados con el quehacer profesional, que con las disciplinas científicas.

Sin embargo, la organización de un colegio for mal a tra vés de una Aso cia ción pro fe sional y el uso del mé to do cien tífico en cada una de las investigacionesmétricas aplicadas, la calidad y objetividad de los datos, métodos y téc ni cas em plea das y el de sa rro llo de in ves tigaciones teóricas, mucho pueden aportar no sólo a es cla re cer la mal lla ma da "cri sis", sino que coad yu va ría a sen tar las ba ses teó ri cas y metodológicas de los estudios métricos de la Información.

No debe ol vi dar se que el sur gi mien to de cada uno de los tér mi nos que hoy se ma ne jan para definir disciplinas o más bien es pecialidades mé tri cas par tie ron del ejer ci cio de fun da mentar la identificación de una serie de trabajo que se distinguían fundamentalmente por la aplicación de métodos y modelos matemáticos a la información documentaria; aplicación que fue varian do, en ob je ti vos y pro pó sitos, en la medidaquefuerondiferenciándose las unidades de observación y por consiguiente las disciplinas científicas y actividades que le dieron origen.

Se ría erróneo pre ten der que el sur gi mien to de la In for má ti ca en Eu ro pa del Este, de la Ciencia de la Información o el de la Cien cia de la Documentación en Norteamérica y España, respec tivamen te, en tre otros tér mi nos utilizados en el in ten to por de fi nir una nue va dis ciplinacien tífica, comore sul ta do del de sarrollo de la ac ti vi dad in for ma ti va en el pe río do de la post gue rra, fue ra a sus ti tuira la Biblio te cología o a con ver tir la en par te de un todo, ello no se ría po si ble por que am bas tie nen te mas y obje to de es tu dios que la di fe ren cian en tre sí, al igual que la Bibliografología y la Archivología.

El comportamientoy de sa rro llo de es tas disci pli nas nos per mi te pen sar que en el proce so de apli ca ción de los mé to dos y mo de los mtemá ti cos y es ta dís ti cos a las ac ti vi da des y objetos de estudio propios de estas disciplinas, como parte de las relaciones interdisciplinarias que se ma ni fies tan en to das las ra mas del saber, bien pue den sur gir es pe cia li da des mé tricas que se diferencien entre sí y puedan aportar nuevos conocimientos a las disciplinas que les dieron origen.

A la luz de este en fo que, sis té mi co por de más, cues ta tra ba jo en ten der que la so lu ción al pro- ble ma, oportuna men te plan tea do por Glan zel y Schoepflin en el citado artículo, ${ }^{(25)}$ pueda re sol ver se con la bús que da de un nom bre para unaes pecialidad odis ci pli naque pre ten daenmarcar tanta diversidad de conocimiento.

Autores como J.M. Russell ${ }^{(33)}$ distinguen la diferencia y a su vez las estrechasrelaciones que exis ten en tre la Bi blio me tría, la In for metría y la Cien cio me tría, as pec tos es tos con los que coincido plenamente, no así cuan do más ade lan te se ña la que la In for me tría y la Bi bliometría deben considerarse subcampos de la Cienciometría; esto sería como postular que la Cien cio lo gía o la Cien cia de la Cien cia pueda contener como subcampos o especialidades a la Bibliografología o a la Ciencia de la Información.

No que da des car ta da la es tre cha re la ción que exis te en tre to das la es pe cia li da des mé tricas, incluyendo la Cienciometría, al igual que entretodaslas disciplinas Bibliológico-Informativas y la Cienciología; incluso el uso común de variable e indicadores entre ellas, pero no debe per der se de vis ta el fin úl ti mo de cada investigación y hacia qué disciplina apuntan y enriquecen sus resultados.

Con el in te rés de mos trar gráfica men te las relaciones entre disciplinas, especialidades y actividadesque se identifican en los estudios mé tri cos de la in for ma ción, se mues tra a continuación el esquemasiguiente.

La de li mi ta ción de es tos te mas de es tu dios, de los ob je ti vos que se per si guen en cada es tu dio métrico de la información y su estrecha relación con las dis ci pli nas cien tí fi cas que en marcan las actividades bibliotecaria, archivística, bibliográficae in for mativa, ha per mi tidola defi ni ción de con cep tos y for mu la ción de pos tu-

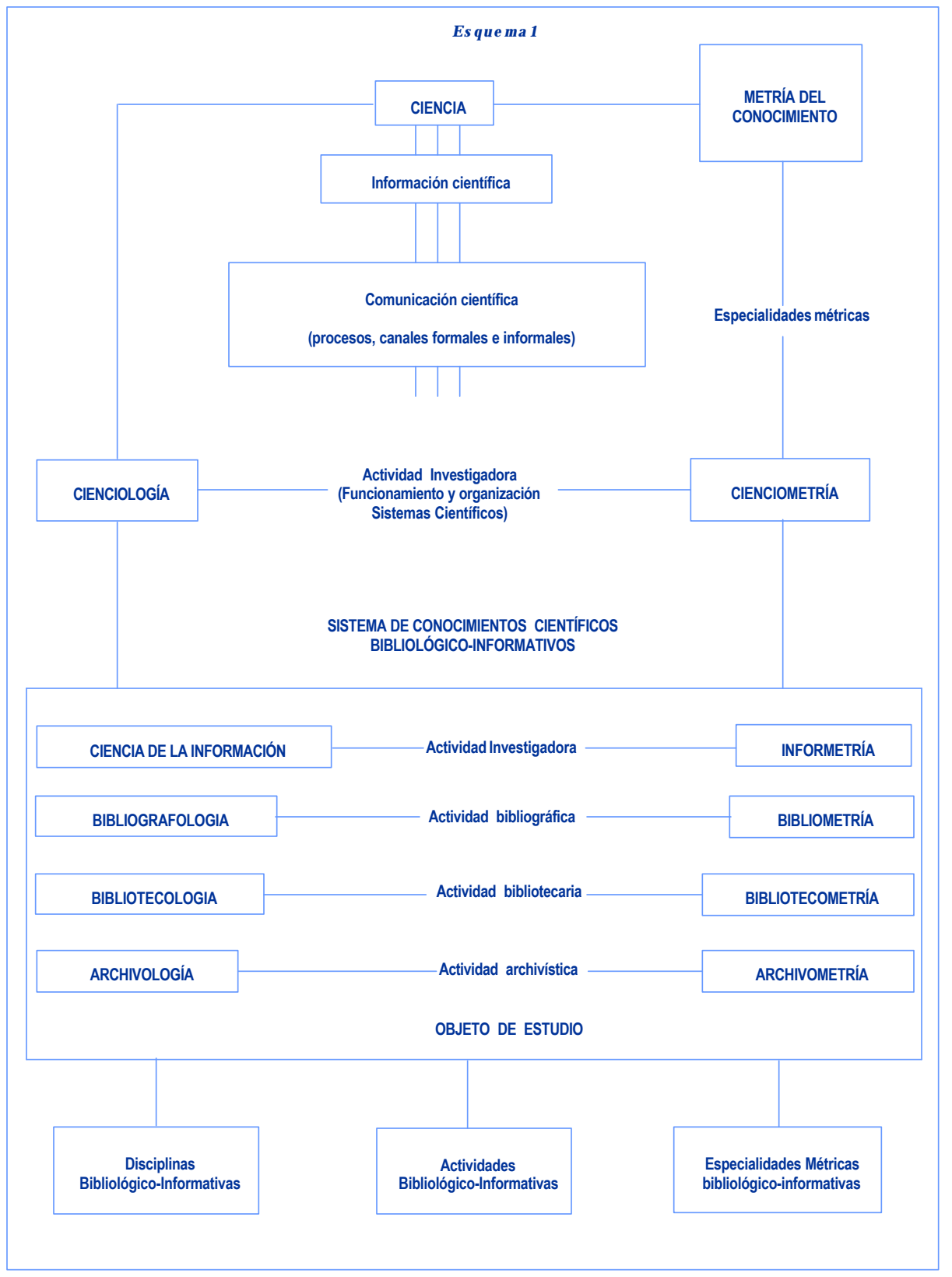


la dos teó ri cos, así como de mo de los ma te máticos que con tribuyen a con for mar un cuer po de co no ci mien tos que cada vez más apun ta al surgimiento de nuevas especialidades, como resultado de la interrelación entre los métodos y modelos matemáticos y estadísticos y un sistema de conocimientos más amplio comoelBibliológico-Informativo o el perteneciente a la Cienciología.

\section{MODELO METODOLOGICO PARALOS ES TUDIOS ME TRI COS DELA I NF ORMACION}

En este con tex to, se mues tra a con ti nua ción la aproximación de un modelo metodológico, el cual pre sen ta los ele men tos bá si cos y sus re laciones a con si de rar en los es tu dios mé tri cos de la in for ma ción que com pren de: el mar co teó ri co-conceptual o sistema de conocimientos en el cual se apo yan el aná li sis cuan ti ta ti vo y cualitativo de la información documentaria; las fuentes que intervienen en el análisis; las unida des bá si cas de aná li sis y ob ser va ción a considerar según el tema u objeto de estudio; las variables seleccionadas según unidades de análi sis; y los mé to dos, mo de los ma te má ti cos y tec nolo gías utili za dos como he rra mien tas en este tipo de estudio.

\section{VA RIA BLES A CON SI DERAR EN EL ANALISISMETRICO DE LA INFORMACION}

En años re cien tes ha sur gi do gran in te rés en el análisis de los Flujos de Información Documentaria de campos interdisciplinarios, a través del aná li sis de las fuen tes de in for ma ción, y en par ticular en su con cen tra ción, dis persión, distribución temática y geográfica, comportamiento de las citas y control bibliográfico.

Ello ha sido po si ble gra cias a la exis ten cia de repertoriosbibliográficos especializados, nacionales e internacionales, en soportes magnéticos u ópticos los cuales compilan parte del producto informativo generado en una rama del saber, período o rea geográfica deter mi na da, di fun di dos a tra vés de servi cios y sis te mas de in for ma ción es pecializados.

Estosrepertorios especializados describenlos documen tos im pre sos o no a par tir de los da tos que los iden ti fi can y que con for man ellla ma do asien to bi blio grá fi co, el cual no sólo orien ta al usua rio del re per to rio en la bús que da de fuentes primarias, sino que además constituyen uni da des bá si cas de análi sis y ob serva ción en la des crip ción de los com po nen tes que ca rac teri zan la es truc tu ra de un de ter mi na do flu jo de informacióndocumentaria.

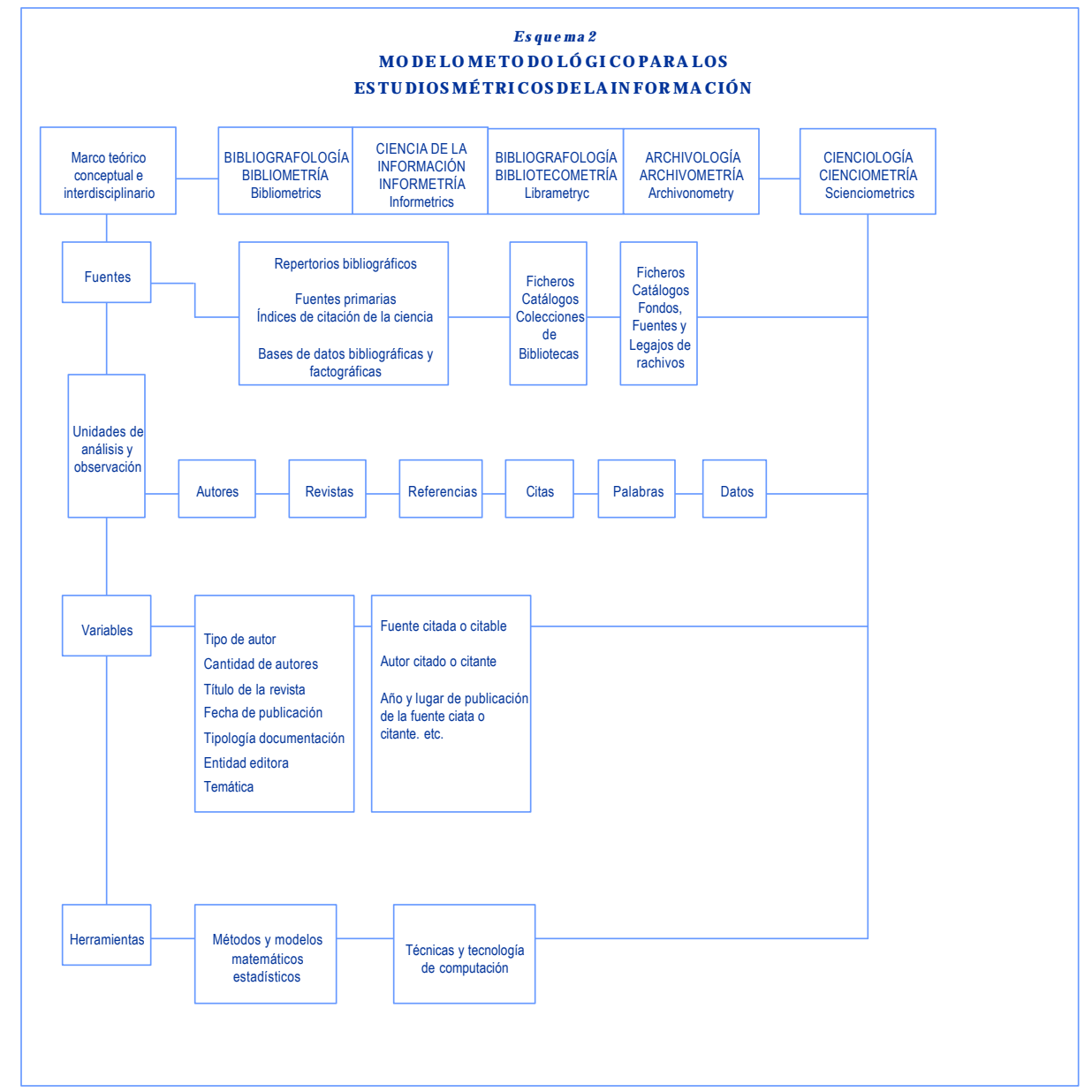

Desde el punto de vista cuantitativo es tas refe ren cias bi blio gráfi cas o uni dad de análi sis y observación, en un estudio bibliométrico, permiten la identificación de variables suscep ti bles decuan tificar por me dios ma nuales o au to má ti cos, lo cual per mi te la ob ten ción de tablas de fre cuen cias en can ti da des ab so lu tas y re la ti vas, que fa ci li tan la apli ca ción de in dicadores, mé to dos y mode los ma te má ti cos en la búsqueda de tendencias o regularidades que conducen al conocimiento del ciclo de crea ción in te lec tual en todo un flu jo de in formacióndocumetaria.

Entre estas va riables se encuen tran:

- Tipo de autor

- Títu lo de la re vis ta

- Cantidad de autores

- Entidad editora

- Lugar de publicación

- Temática

- Fecha de publicación

- Cubrimiento geográfico

- Tipología documentaria

- Idioma, etc
Cuando la uni dad bá si ca de aná li sis y ob serva ción, o sea, la des crip ción bi blio grá fi ca no es analizada en un repertorio bibliográfico, sino como parte de un documento primario, en fun ción de una re fe ren cia o de una cita, pode mos agre gar al es tu dio mé tri co otras va riables tales como:

- Título de la fuente citada o citante

- Autorcitadoocitan te

- Año de pu bli ca ción de la fuen te ci ta da o citante, entre otras.

En este sen ti do vale des ta car las de fi niciones de cita y referencia se gún E. Garfield:(34)

Citas: Son el reco noci mien to que un documento recibe de otro, indica la influen cia oim pac to cien tífico de un trabajo o resultado dado.

REFERENCIAS: Son el reconocimiento que hace un do cu men to a otro y refleja los antecedentes de un trabajo.

Este en fo que con cep tual cons ti tu ye la mé du la metodológica sobre la cual se sus ten ta el sis- 
te ma de In di ces de Ci tas de la Cien cia (Science Citation Index, Journal Citation Reports, etc.), lidereado por este autor, sirve de base tam bién para el cál cu lo de in di ca do res cien tífi cos ta les como el Fac tor de Im pac to (Impact Factor), Indice de Inmediatez o de prontitud de Cita (ImmediacyIndex), Vida Me dia ( Hal$f$-Life), entre otros.

Las ci tas, por su pues to, pre ci san Krau ze y Hillin ger(1971), ci ta dos por Lo pez Pi ñe ro en su obra, ${ }^{(4)}$ las recibe una publicación de otras posteriores, mientras que las referencias las hace una pu bli ca ción de otras an te rio res; más adelante señala que los referidos autores insisten en que el número de citas de trabajos escritos en un momento determinado es distinto al de las referencias contenidas en los mismos, puesto que las dos cantidades son numéricamente diferentes.

Otraposibilidad de análi sis de las fuen tes de información, y más específicamente de los docu men tos pri marios lo cons ti tu ye su análisis de contenido el cual permite (tomando como unidad de análisis y observación a la pa la bra) la apli ca ción de la dis tri bu ción de G. Zipf,(35) modelo éste que posibilita, a través del análi sis cuan ti ta ti vo de fre cuen cia de apari ción de pa la bras en un tex to dado, es ta ble cer los ne xos y re la ciones do cu men ta les des de el punto de vista de contenido lingüístico o interdisciplinario, de una fuentes de información pri ma ria, de un au tor o de una dis ci plina determinada.

En cada caso se originanvariablesdiferentes y se cuantifican con objetivos también diferentes, por ejemplo:

- El análisis cuantitativode las variables en los repertorios bibliográficos especializados nos permite conocer sobre las tendencias y regularidades de la producción científica en un flujode in for mación documentaria de una determinadatemática.

- La con tabi lidad de variables en las re fe rencias relacionadas en los documen tos primarios nos permite conocer el comporta mien to del flu jo de in for ma ción re fe ren ciado y el grado de envejecimiento u obsolecencia de los documentos que integran el flujo.

- El aná li sis de la fre cuen cia de apa ri ción de las va ria bles en una o más ci tas nos in di can el comportamiento de la influencia o impacto científico de uno o varios documentos pertenecientes a uno o varios autores, instituciones o países.

La dis tribu ción de pa la bras de acuer do con su frecuencia de aparición en un texto determinado nos permi te conocerla prevalencia o au to ri dad de és tas para ser in clui das en índices, lenguajescon troladosoevaluación descriptiva de los ficheros y catálogos de biblioteca.
En el pri mer caso, los asien tos bi bliográ fi cos de los re per to rios o los da tos al ma ce na dos en una base fac to gráfica nos re ve lan las re gu la ri dades de la información científica en una rama del saber específica; en el segundo, las re fe ren cias de los docu men tos pri ma rios permitenidentificarnexos documen tarios, obsolescencia y antecedentes de la producción científica; en el tercero, el análisis de citas ofrece el impacto, la visibilidad o reconocimiento que un documento, autor, institución o país re ci be de otro; y el cuar to per mi te la generación de índices, catálogos de autoridad, así como identificar los nexos lingüísticos y de con te nidoin ter disci plina rio que exis te entre documentos.

El es que ma que se mues tra a con ti nua ción sirvió de base para la dis cu sión de un tra ba jo ante rior re la cio na do con los prin ci pios me to dológicos en el análisis de los repertorios bibliográficos, S. Gorbea, ${ }^{(36)}$ e ilus tra los casos antes descritos.

En cada caso el resultado obtenido surge como consecuencia del análisis de las mismas variables documentarias en diferentes fuen tes de in for ma ción, lo que per mi te la obten ción de re sul ta dos tam bién di fe ren tes, utilizando como herramientas, diferentes métodos y modelos matemáticos.

\section{PRINCIPA LES METODOS YMODELOS MA TE MA TI COS APLICADOS ENLOS ES TU DIOS ME TRI COS DE LA INFORMACION}

Para determinar el comportamiento de las tendencias y regularidades de la información científica y técnica se utiliza una gama muy variada de métodos y modelos matemáticos, en tre los más clási cos se gún re gu la ri da des tenemos los siguientes:

\section{CONSIDERACIONESGENERALES}

Baste una sucinta identificación de las variables que utilizan estos métodos y modelos matemáticos para poder percibir que con independencia de los resultados que se obtengan "los cua les es tán de ter mi na dos por el análi sis y en fo que de sus va riables" to man como base o fuen te de in for ma ción fun da men tal a la información registrada en documentos prima rios, re per to rios biblio grá fi cos o a la in forma ción que se ob tie ne du ran te el pro ce so del análi sis de con te ni do inhe ren te a la com pi lación de éstos.

LaCien cio me tría es una es pe cia li dad mé trica de la Cien cio lo gía que uti li za, en la me tría del conocimiento, variables e indicadores métricos de la in for ma ción do cu men ta ria porconstituir las publicaciones científicas el canal más utilizado en la transmisión del conocimiento científico, pero no por ello debe ser considerada como disciplina que englobe la to ta li dad de es tu dios mé tri cos que se rea li zan con la in for ma ción do cu men taria.

En los es tu dios mé tri cos de la in for ma ción la de finición de fac to res ta les como: el obje to de estudio, el procedimien toutilizado, lasunidades de ob servación y el ob je ti vo cien tífi co del análisis, dentro del denominado sistema de conocimientos científicos Bibliológico-Informativo no sólo contribuye al enriquecimien to de este sis te ma de co no ci mien tos sino que tam bién pro pi cia el sur gi mien to y con so-

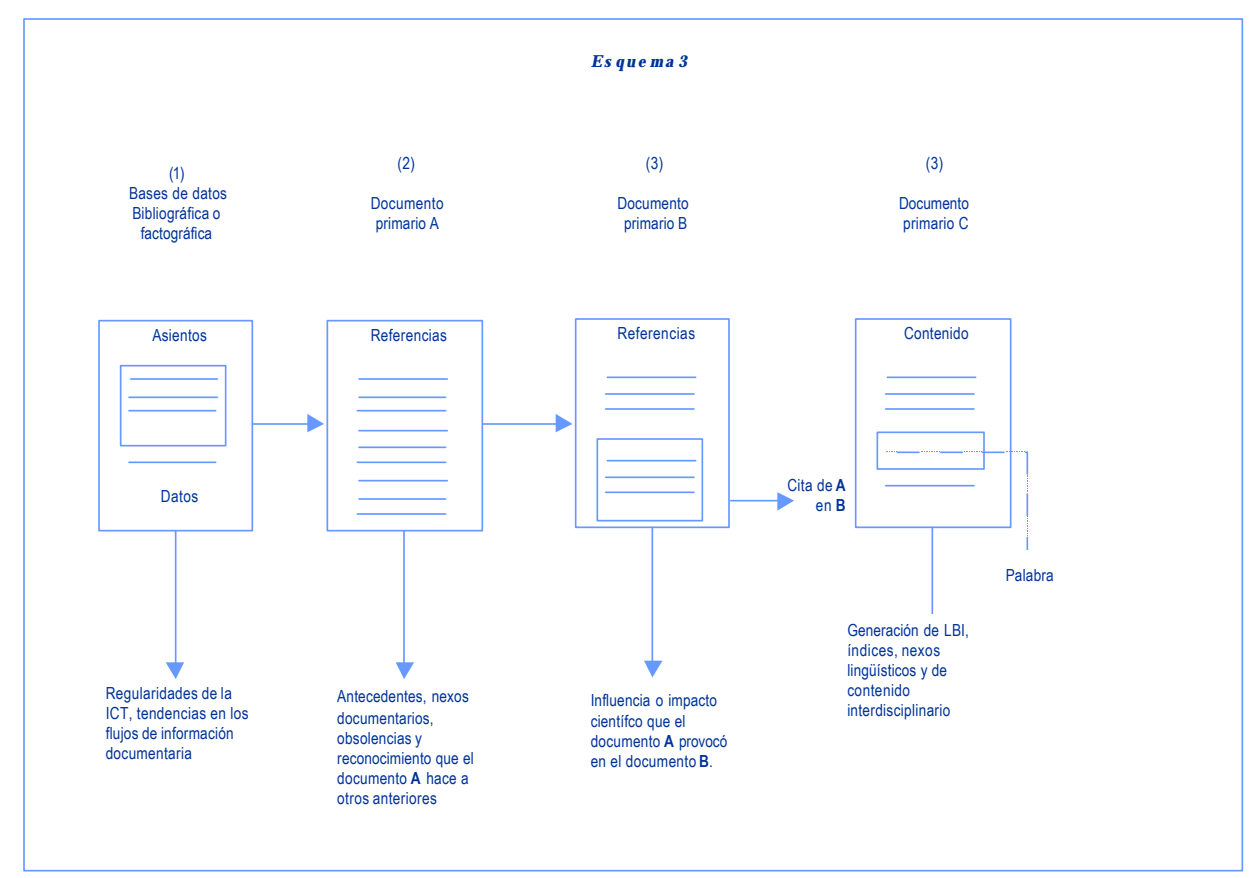


lidación de las denominadas especialidades mé tri cas de la in for ma ción.

Resulta imposible pretender que toda la diver si dad de es tu dios mé tri cos a los que es sometidalain for mación documen taria, en objetivos, fines y relaciones disciplinarias diferentes, pueda ser enmarcada en una sola especialidad, sin considerar que cada aporte que se realiza en este tipo de estudio, puede utilizarunidades de observación, in dicadores y variables diferen tes las cua les de ter minanel fin último de la investigación y aportan en cada caso información-conocimiento a una gama muy di versade dis ci plinas cien tíficas.

Se ría más con ve nien te di vi dir este tipo de estu dio en es pe cia li da des mé tricas que evi ten la de no mi na da "cri sis", a la cual se lle ga al preten der, con un en fo que to ta li ta rio, atribuir le a una sola es pe cia li dad mé tri ca el ca rác ter rector sobre las demás que, dicho sea de paso, surgieron por la observación de uno de los procesosnaturales deldesarrollocientíficode nuestro tiempo, nos referimos al proceso de diferenciación de la Ciencia.

\section{Prquame 4

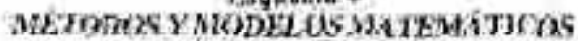 SEGEXER NHACACION}

\section{Repulariviates do la IC.T, Analisis $y$ Icadencias}

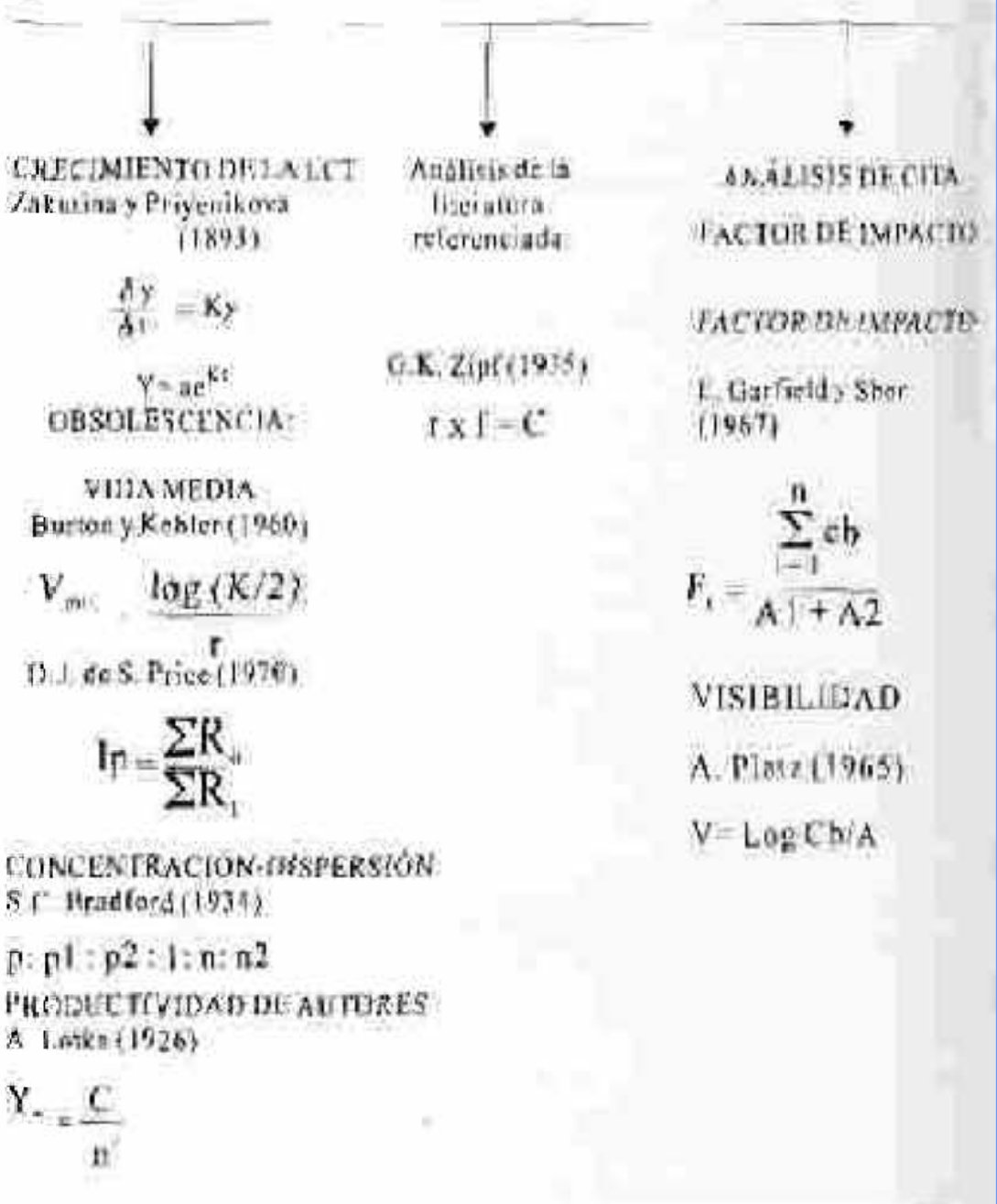

\section{REFERENCIAS}

1 Mijailov, A.I., A.I. Chiornii y R.S. Guilarievskii. Fundamentos de la Informática._La Ha ba na:IDICT, Aca de mia de Cien cias de Cuba, 1973.— 2 t

2. Sokolov, A.U. Sistema de ciencias comunicativo-informativas. Actualidades de la información cien tífica y téc ni ca (La Ha ba na)3(128):5-15, 1986

3. Se tién Que sa da, E. y S. Gor bea Por tal.-De la Biblio te cología al Sis te ma de Conocimientos Científicos Bibliológico-Informativo.-InvestigaciónBibliotecológica(México) 8(16):21-25, enero-junio, 1994

4. López Pi ñe ro, J. Ma.-Análi sis es ta dís ti co y so cio mé tri co de la li te ra tu ra científica.-Cua der nos De Do cu men ta ción E In for ma ción Me di ca I. (Va len cia), 1972 , pp. 82 . 
5. Pritchard, A. and G.R. Wittig.- Bibliometrics: a Bibliography and Index, Vol.1:1874-1959. (Watford: ALLM Book, 1981)

6. Hjer ppe, R. - A Bi blio graphy of Bi blio me trics and Citation In de xing and Analysis .- Stockholm: The Royal Institute of Technology, Library, 1980.- (Report TRITA-LIB-2013).

7. Hjer ppe, R. "Supple ment to a Bi blio graphy of Bi blio me trics and Ci ta tion Analysis." Scientometrics (Budapest) 4:241-281, 1982

8. Khurshid, Anwery Har deo Sa hai. "Bi blio me tric, Scien to me tric and In for me tric Distri bu tions and Laws: A Se lec ted Bi blio graphy." en Fo rum Inf. And Do cum. 16(2):18-29, April, 1991.

(9) Schubert, A.- Quantitative Studies of Science: A Current Bibliography. Scien to me trics (Bu da pest) 5:125-133;397-403, 1983.

- Scientometrics (Budapest) 6:55-59;197-202;359-367,1984

- Scientometrics (Budapest) 7:111-117, 1985

- Scientometrics (Budapest) 7:137-140, 1985

- Scientometrics (Budapest) 9:293-34, 1986

(10) Setién Quesada,E.y S. Gorbea Portal-_ Con cep tos mé tri cos en las dis ci pli nas biblioteco-informativas.- Actualidades De La Información Científica Y Técnica (La Habana) (No. es pe cial, 156) : 3-17, 1990.

(11) Nacke, O.- In for me tría: Un nue vo nom bre para una nue va dis ci plina. De fi nición, es ta do de la cien cia y prin ci pios de de sa rrollo.- Re vis ta Es pa ño la De Documentación Científica (Madrid) 6(3):183-203, 1983

Re don do Botella,.- Una apli ca ción de las ma te má ti cas a la Cien cia de la In forma ción: Ca te go ri za ción de la ley de di fe ren cia ción de las cien cias / Ig na cio García Díaz y Leticia Espinosa Cruz - Actualidades De La información Científica Y Técnica (La Habana) 1(78):1-29, ene ro, 1979

(13) Morales Morejón, M._-El con cep to mé tri co en la In for mática: In for me tría.Actualidades De La información Científica Y Técnica (La Habana) 1(120):33-85, mar zo, 1985

Cambell, F.- The Theory of the National and the International Bibliography.-Lon don, 1896.- pp. 86-97 (Ci ta do por Re don do Bo tel la, L. et. all.)

(15) Cole, F.J. and N.B. Ea les.- The his tory of com pa ra ti ve ana tomy. Part I: A statis ti cal Analysis of the li te ra tu re.-Scien ce Pro gress 11(44):578-596, 1917 (Citado por O.Nacke)

(16) Hul me, E.W.- Sta tis ti cal Bi blio graphy in re la tion to the Growth of mo dern civilization.- London, 1923 (Citado por O.Nacke)

(17) Ranganathan, S.R.- Librametry and its scope, In: Subject analysis for document finding system. Quantification and librametric studies. Management of tras la tion service. In dian Sta tis ti cal Ins ti tu te (Bangla do re, In dia)/Documentation Research Training Centre:Indian Statis ticalInstitute, 969(DRTC Annual Seminar, 7, Vol.1: Papers) (Citado por O. Nacke)

(18) Morales Morejón, M. - La In for me tría y su im por tan cia. En: Se mi na rio In trodu ción a la In for me tría: Análi sis de los Flu jos In for ma cio na les y eva lua ción e las fuentes de información (Compilación). - La Habana: IDICT-SOCICT/CI, 1988.- pp. 1-27 
(19) Pritchard, A.- Statistical Bibliography or Bibliometrics?.- Journal Of Docuumentation (London) 25(4):348-349, de cember, 1969.

(20) Ravichandra Rao, Inna Ke dage.-Quan ti ta ti ve Methods for Li brary and In formation Science.—Banga lore, In dia : John Wi ley \& Sons, [1983].—272 p.

(21) Egghe, L. and R. Rousseau. - Introduction to Informetrics: Quantitative Methods in Library, Documentation and Information Science.- Netherlands: El se vier, 1990.-450 p.

Broo kes, B.C. Biblio-, Scien to-, In for-Me trics??? What are Tal king About?.pp 31- 43 In: In for me trics 89/90. Se lec tion of Pa pers Sub mi tted for Se cond InternationalConference on Bibliometrics, Scien to me trics and In for me trics./ Edited by L. Egghe and R. Rousseau.— Nether lands, 1990.—401 p.

Glanzel, W. and U. Schoepflin. Little Scientometrics, Big Scientometrics.... and Beyound. Scientometrics (Budapest) 30(2-3):375-384, 1994

Krauskopf, M. Epistetometria, a Term Contributing to Express the Meaning and Po ten tial Metho do lo gies of Scien to me trics in Spa nish Spea king Country. Scien to me trics (Bu da pest) 30(2-3):425-428, 1994

Bonitz, M. Pro mo ting Scien to me trics by In ter na tio nal Schools Scien to me trics (Budapest) 30(2-3):451-453,1994

Rousseau, R. Si milarities Bet ween In for me trics and Eco no me trics. Scien to metrics (Budapest) 30(2-3):385-387, 1994

Egghe, L. Little Science, Big Science.... and Beyond. Scientometrics (Budapest) 30(2-3):389-392, 1994

Eto, H. Quality Assessment of Science Information Research and Service. Scien to me trics (Bu da pest) 30(2-3):429-432,1994

Brookstein, A. Scien to me trics: New Oppor tu nities. Scien to me trics (Bu dapest) $30(2-3): 455-460,1994$

McGrath, W. E. Little Scientometrics, Big Scientometrics.... and Beyound. Scien to me trics (Bu da pest) 30(2-3):439-442, 1994

Russell, Jane M. Back to the Fu tu re for In for me trics. Scien to me trics (Bu da pest) 30(2-3):439-442, 1994

Institute for Scientific Information: Terminology and Definitions. ScienceCitation Index. Fi la del fia, 1988 Vol 1. IC.

Zipf, G.K.- Human Behavior and the principle of least effort.- Cambridge Mass.: Addi son-Wes ley, 1949

Gor bea Por tal, S.- Aplica ción de los mé to dos y mo de los ma te máti cos al análisis de los re per to rios bi blio gráficos. — La Habana, 1991.—22 p. (Ponencia presentada en el 1er. Simposio de Bibliografía, Biblioteca Nacional, La Habana, junio de 1991) 